\title{
determinación de las proporciones de escoria y cemento o cal en una mezcla de estos materiales
}

\author{
F. S. FULTON \\ Director Portland Cement Institute, South Africa \\ Cement and Lime Manufacture $n^{\circ} 3$, mayo 1969, págs. 41-47
}

\begin{abstract}
En Africa del Sur es práctica común en muchos lugares de construcción emplear mezclas de cemento portland y escoria de horno alto granulada molida, para la fabricación de hormigón. Las propiedades hidráulicas de la escoria molida son relativamente débiles, pero, en una mezcla de cemento portland y escoria, el hidróxido cálcico formado en la hidratación del cemento parece activar la escoria. Supuesto que la mezcla es adecuada, la mezcla in situ del cemento portland ordinario y la escoria de horno alto molida en proporciones iguales en peso produce un material que tiene propiedades similares a la de los cementos portland de horno alto.
\end{abstract}

Usualmente, la mezcla se lleva a cabo en obra simultáneamente con, y como parte de, la operación de amasado del hormigón. En la práctica un saco de $43 \mathrm{~kg}$ de escoria molida es sustituido en la amasadora por un saco de $43 \mathrm{~kg}$ de cemento portland, no siendo necesario ampliar el tiempo de amasado normal. En las grandes obras, la escoria y el cemento se suministran generalmente a granel, y estos materiales se mezclan antes de su alimentación a la planta de hormigonado.

Como medida de control, y más concretamente como una comprobación de la eficiencia de la planta de mezclado, es necesario ser capaces de determinar la exactitud de las proporciones de cemento y escoria molida en la mezcla. Se han desarrollado un número de métodos, que llevan consigo la difracción de rayos X (3), el análisis térmico diferencial (4) o la determinación de sulfuros (5). Las desventajas de estos métodos son los aparatos caros que requieren, la necesidad de la interpretación de resultados por expertos y un grado de exactitud más bien bajo.

El principio del método propuesto en este artículo, se basa en el hecho de que, si hay siempre disponible agua de curado para la pasta de cemento fresca, se absorbe continuamente agua por el cemento que hidrata. La cantidad de agua es una medida directa de la reducción en volumen del sistema de la pasta y se aproxima a una función lineal de la cantidad de agua no evaporable o "combinada" contenida en ella.

De acuerdo con Powers y Brownyard (1) $W_{a}=k \cdot W_{n}$, donde $W_{a}$ es el peso de agua absorbida en un estado de hidratación dado; $W_{n}$, el peso de agua no evaporable o "combinada" en esta fase de la hidratación, y $k$ es una constante que depende del cemento particular contenido en la pasta.

El autor (2) ha demostrado que esta expresión sería más correcta si $W_{\mathrm{a}}=A+B \cdot W_{\mathrm{n}}$, donde $A$ y $B$ son constantes para un cemento particular. 
En una mezcla de escoria molida y cemento portland, la cantidad de agua "combinada" en cualquier fase de la hidratación depende de las características de ambos (cemento y escoria) y de su proporción en la mezcla. En consecuencia, si mediante ensayo se establecen las velocidades de absorción de mezclas de escoria molida y cemento portland hechas en proporciones conocidas, entonces la cantidad de agua absorbida en el mismo período por una mezcla en proporciones desconocidas puede emplearse para establecer las cantidades de ambos componentes en la citada mezcla.

El método se ha desarrollado específicamente para el uso de mezclas con escoria, pero es probable que pueda aplicarse también a mezclas de cemento y otros materiales que tengan propiedades puzolánicas. La principal desventaja reside en que, para llevar a cabo el ensayo, debe de disponerse de muestras del cemento portland y de escoria empleados en la mezcla. Esto es de pocas consecuencias en un gran proyecto donde la toma de muestras continua de silos a granel no ofrece dificultades.

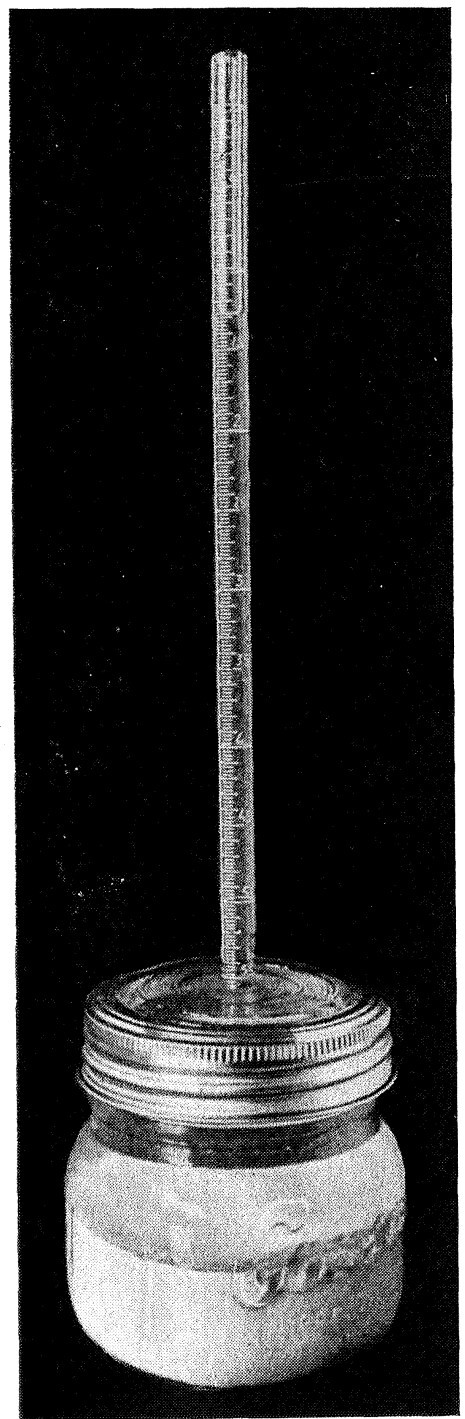

Fig. 1.-Conjunto de la bureta de absorción: ensayo en curso.

\section{APARATO}

La única pieza esencial, aparte de una balanza, es la bureta de absorción mostrada en las figuras 1 y 2 . El frasco consiste en un tarro provisto de la placa de vidrio de cobertura, casquete metálico y arandela de goma. La cubierta metálica está perforada para recibir una bureta, haciéndose el agujero de paso impermeable con resina epoxi. La ventaja de este tipo de aparato estriba en que los tarros son tan baratos que pueden desecharse después de su uso, en tanto que la placa de cobertura y la bureta pueden volverse a usar cualquier número de veces.

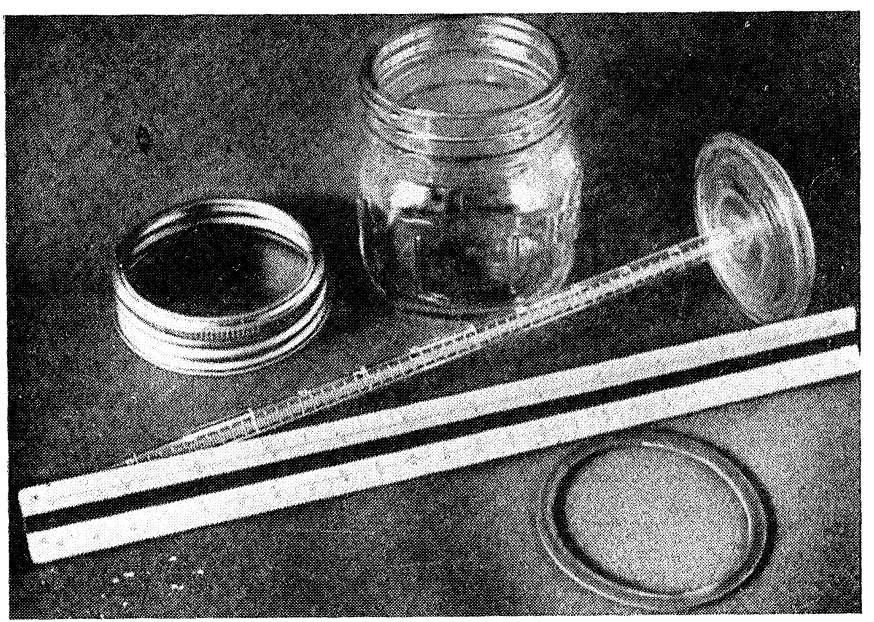

Fig. 2.-Componentes de la bureta de absorción. 
Es conveniente emplear grandes muestras de pasta hasta unos $400 \mathrm{cc}$. Aun con buretas graduadas sólo a $0,1 \mathrm{ml}$, la cantidad de agua absorbida puede leerse con exactitud hasta $0,1 \mathrm{mg} / \mathrm{g}$ de material conglomerante.

Para el fin propuesto con este ensayo es más adecuada una bureta de $6 \mathrm{ml}$ graduada a $0,025 \mathrm{ml}$. Para ensayos que se extienden a períodos mayores de 24 horas, es preferible una bureta de $20 \mathrm{ml}$. Es importante que la bureta esté completamente limpia, empleando un trozo de lana de algodón después de cada ensayo. Si esto no crea dificultades puede experimentarse leyendo el menisco.

\section{PROCEDIMIENTO}

$400 \mathrm{~g}$ de conglomerante se mezclan con $160 \mathrm{~g}$ de agua destilada. El amasado se lleva a cabo a mano durante 4 minutos en un tazón de esmalte o acero inoxidable. Para trabajo preciso de laboratorio es conveniente una temperatura y humedad controlada. La pasta entonces se vierte en el tarro y se consolida sacudiéndolo para expulsar las burbujas de aire. Inmediatamente después, el tarro se llena con agua, empleando una pequeña jeringa de forma que se eviten perturbaciones en la superficie de la pasta. La operación se completa colocando todo el aparato en un tanque o artesa llena de agua, cerrando cuidadosamente la cubierta de la bureta, el casquillo metálico y la arandela de goma para excluir el aire. A la salida del tanque, se cierra perfectamente el casquillo metálico y la bureta se llena hasta la marca de 0,5 $\mathrm{ml}$. Desgraciadamente el apretado del casquillo requiere dos operadores. Finalmente, se colocan unas cinco gotas de aceite sobre la superficie del agua en la bureta para prevenir la evaporación, y el aparato se coloca en un armario de conservación. Para un trabajo de laboratorio exacto es necesario controlar las condiciones de temperatura durante la conservación.

La primera lectura de la bureta se toma exactamente 2 horas después del principio del amasado y ésta se considera como lectura cero para el ensayo. La lectura final de cada muestra se toma exactamente 21 horas después de la adición del agua de amasado. Pueden elegirse otros períodos de conservación, pero el de 21 horas fue bien en los laboratorios de este Instituto. Se prepararon cinco muestras por la tarde, de forma que la lectura origen de la última muestra se tomase justo antes del cierre de los laboratorios (a las cinco). Esto quiere decir que el ensayo tiene lugar durante la noche y los resultados se recogen en la mañana del día siguiente.

\section{RESULTADOS}

Los ensayos de absorción durante 21 horas de varias mezclas de escoria molida y un cemento portland se representan en la curva ASB de la figura 3, que es típica de los resultados obtenidos. Las absorciones se caiculan simplemente, dividiendo la caída en el menisco de la bureta por el peso de escoria y cemento combinados en la pasta, expresándose los resultados en $\mathrm{mg} / \mathrm{g}$ de conglomerante.

Puede señalarse que con proporciones cemento: escoria entre 40:60 y 60:40, la curva de absorción es prácticamente una línea recta. Por lo tanto, para determinar las proporciones de una mezcla, es sólo necesario hacer mezclas de los materiales disponibles en dichas proporciones y comparar sus absorciones a 21 horas con la de la mezcla desconocida. Las proporciones en la última pueden obtenerse por interpolación directa. Para determinaciones más exactas es conveniente ensayar mayor número de muestras y más próximas entre sí. 
La tabla I muestra los resultados obtenidos para mezclas de cinco cementos normales y dos escorias molidas de distinto origen. Además, como medida de control, se hicieron las mezclas correspondientes de los cementos con un material inerte, por ejemplo polvo de mármol molido a la finura de cemento.

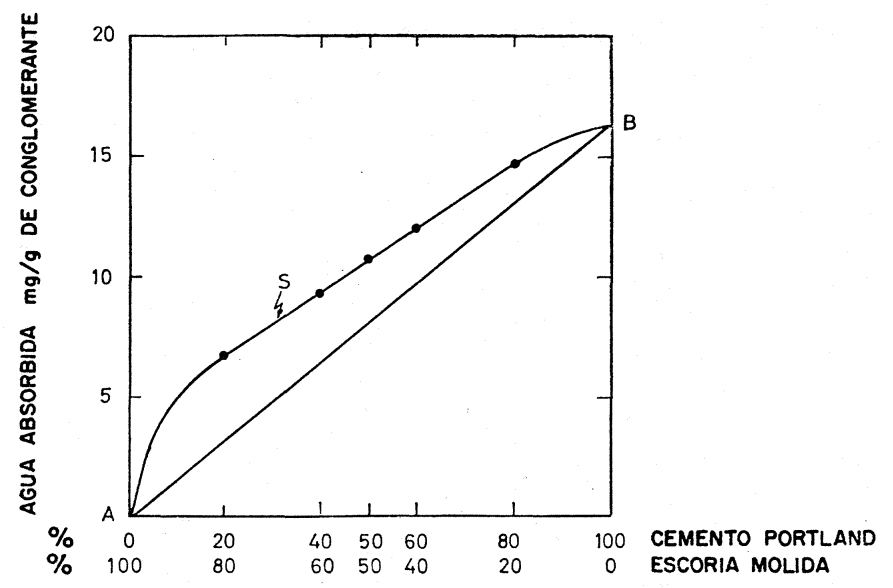

Fig. 3.-Curva de absorción típica para mezcla de cemento port and y escoria de horno alto mo-

T A B L A I

Proporciones reales y determinadas para mezclas de cementos portland, escorias molidas $y$ material inerte

\begin{tabular}{|c|c|c|c|c|c|c|c|c|c|c|c|}
\hline \multirow{3}{*}{ Cemento } & \multirow{3}{*}{ Escoria } & \multicolumn{10}{|c|}{ Proporciones reales } \\
\hline & & \multicolumn{2}{|c|}{$55 / 45$} & \multicolumn{2}{|c|}{$52,5 / 47,5$} & \multicolumn{2}{|c|}{$50 / 50$} & \multicolumn{2}{|c|}{$47,5 / 52,5$} & \multicolumn{2}{|c|}{$45 / 55$} \\
\hline & & $W_{\mathrm{a}}$ & Det. $\%$ & $W_{\mathrm{a}}$ & Det. \% & $W_{\mathrm{a}}$ & Det. \% & $W_{\mathrm{a}}$ & Det. $\%$ & $W_{\mathrm{a}}$ & Det. \% \\
\hline \multirow[t]{3}{*}{03} & 1 & 11,08 & 44,57 & 10,60 & 48,05 & 10,30 & 49,98 & 9,97 & 52,62 & 9,67 & 54,78 \\
\hline & 2 & 11,27 & 44,76 & 10,82 & 47,87 & 10,53 & 49,81 & 10,10 & 52,76 & 9,80 & 54,81 \\
\hline & inerte & 11,32 & 44,98 & 10,82 & 47,71 & 10,42 & 49,89 & 10,00 & 52,17 & 9,43 & 55,25 \\
\hline \multirow[t]{3}{*}{04} & 1 & 9,25 & 45,23 & 9,03 & 47,30 & 8,73 & 50,16 & 8,57 & 51,72 & 8,17 & 55,53 \\
\hline & 2 & 9,55 & 44,75 & 9,18 & 48,20 & 9,03 & 49,62 & 8,77 & 52,14 & 8,43 & 55,28 \\
\hline & inerte & 9,32 & 44,84 & 8,95 & 47,71 & 8,68 & 49,78 & 8,27 & 52,99 & 8,05 & 54,70 \\
\hline \multirow[t]{3}{*}{08} & 1 & 10,27 & 44,96 & 10,01 & 47,58 & 9,80 & 49,70 & 9,47 & 53,02 & 9,30 & 54,74 \\
\hline & 2 & 11,90 & 45,47 & 11,67 & 47,37 & 11,40 & 49,60 & 11,07 & 52,36 & 10,70 & 55,39 \\
\hline & inerte & 10,56 & 45,05 & 10,30 & 47,33 & 9,92 & 50,65 & 9,82 & 51,52 & 9,37 & 55,45 \\
\hline \multirow[t]{3}{*}{09} & 1. & 10,60 & 44,78 & 10,26 & 47,44 & 9,85 & 50,64 & 9,64 & 52,28 & 9,31 & 54,86 \\
\hline & 2 & 11,16 & 45,03 & 10,86 & 47,62 & 10,60 & 49,86 & 10,32 & 52,28 & 9,98 & 55,21 \\
\hline & inerte & 11,24 & 44,93 & 10,82 & 47,43 & 10,35 & 50,24 & 9,97 & 52,51 & 9,57 & 54,89 \\
\hline \multirow[t]{3}{*}{010} & 1 & 10,37 & 44,92 & 10,08 & 47,33 & 9,73 & 50,25 & 9,42 & 52,83 & 9,20 & 54,67 \\
\hline & 2 & 11.08 & $4 \mathfrak{5 , 4 3}$ & 10,92 & 46,98 & 10,58 & 50,27 & 10,42 & 51,82 & 10,04 & 55,50 \\
\hline & inerte & 11,05 & 44,80 & 10,63 & 47,80 & $*$ & $*$ & 9,99 & 52,37 & 9,62 & 55,02 \\
\hline
\end{tabular}

* Material insuficiente. 
Aunque las absorciones de agua determinadas para estas últimas mezclas se corrigieron para la absorción del polvo de mármol sin aditivo, en casi todos los casos los resultados muestran valores de $W_{\text {a }}$ superiores a los obtenidos para las correspondientes mezclas que contienen escoria $\mathrm{n}^{\circ}$ 1. Esto puede señalar alguna pequeña reacción entre el mármol, y el cemento, como ya han señalado algunas autoridades en la materia.

\section{CONCLUSIONES}

1. El ensayo es extremadamente simple comparado con los actuales métodos químicos $\mathrm{u}$ otros métodos.

2. El aparato es simple y barato, y el ensayo puede hacerse confidencialmente por un técnico de laboratorio sin entrenamiento alguno.

3. El ensayo muestra que el error en la estimación de las proporciones es normalmente menor del $0,5 \%$.

4. El ensayo es muy reproducible y evita un número de variaciones indeseables inherentes a otros ensayos, principalmente las debidas a la eficiencia del operador, compactación, uso de áridos, etc.

5. El ensayo puede realizarse con buena precisión a pie de obra. Supuesto que no hay grandes cambios de temperatura en el intervalo de 40 minutos entre preparar la primera y la tercera probeta, o en tomar las correspondientes lecturas de bureta, el control de la temperatura no es de mucha importancia. Si las probetas se conservan juntas, cualquier cambio en la temperatura ambiente afectará a las tres en la misma forma. La humedad no es importante, a menos que surjan cambios muy grandes en el corto período de amasado de las tres muestras.

6. El ensayo ofrece un medio de modificar mezclas de hormigón como consecuencia de cambios en las características del cemento o de la escoria dentro de 21 horas después de recibir en obra estos materiales.

7. Parece que la contribución de la escoria molida a la absorción de agua, y consecuentemente a la resistencia de la mezcla, es despreciable al menos hasta la edad de 21 horas.

\section{MEZCLAS DE CAL HIDRATADA Y ESCORIA MOLIDA}

En Africa del Sur, las mezclas de cal hidratada y escoria molida se emplean frecuentemente para la estabilización de bases de carreteras. La cal y la escoria se suministran generalmente a granel y se mezclan antes de su aplicación en un mezclador portátil especialmente proyectado. El problema además es el de la determinación de las proporciones exactas de los dos materiales en la mezcla.

El método ya descrito en este artículo es totalmente adecuado para este fin. Desgraciadamente la mezcla comúnmente empleada es 2 partes, en peso, de escoria y una parte, en peso, de cal. Como puede verse por la figura 4, las curvas de absorción de agua para mezclas de aproximadamente estas proporciones son más bien planas y no es posible un alto grado de precisión. La dificultad se sobrelleva componiendo mezclas de escoria y cal en proporciones conocidas para "abarcar" la mezcla desconocida. Estas mezclas se amasan íntimamente con cantidades iguales en peso de cal hidratada. De esta forma es posible trabajar en el intervalo de contenidos de cal caracterizado por el cambio de pendiente de la curva de absorción mostrada en la figura 4. La reconversión a las mezclas originales es materia de simple aritmética. 
Los resultados de una serie limitada de ensayos se muestran en la Tabla II. La dilución de las mezclas originales con cal introducía un grado de error que, teniendo en cuenta los fines de la mezcla es, no obstante, tolerable. Debe, sin embargo, señalarse que es necesario usar una relación agua: material conglomerante de 0,75 en peso, para obtener una pasta suficientemente fluida y permitir una fácil compactación en el tarro.

\section{T A B L A II}

Proporciones reales y determinadas de mezclas de escorias molidas y cal hidratada.

\begin{tabular}{|c|cc|cc|}
\hline \multirow{2}{*}{$\begin{array}{c}\text { Contenido real } \\
\text { de cal } \\
(\% \text { en peso })\end{array}$} & \multicolumn{2}{|c|}{ Escoria 1 } & \multicolumn{2}{|c|}{ Escoria 2} \\
\cline { 2 - 5 } \cline { 3 - 5 } & $W_{\mathrm{a}}$ & Det. $\%$ & $W_{\mathrm{a}}$ & Det. \% \\
\cline { 2 - 5 } 20 & 2,80 & 21,58 & 6,98 & 20,09 \\
22,5 & 2,69 & 22,12 & - & -- \\
27 & 2,62 & 26,44 & 6,55 & 26,58 \\
30 & - & - & 6,43 & 27,85 \\
32 & 2,24 & 32,80 & - & - \\
34 & 2,36 & 33,80 & 6,03 & 33,12 \\
36 & - & - & 5,85 & 35,38 \\
38 & 2,04 & 37,55 & - & - \\
40 & 2,09 & 41,58 & 5,43 & 40,76 \\
42 & - & - & 5,23 & 43,43 \\
\hline
\end{tabular}

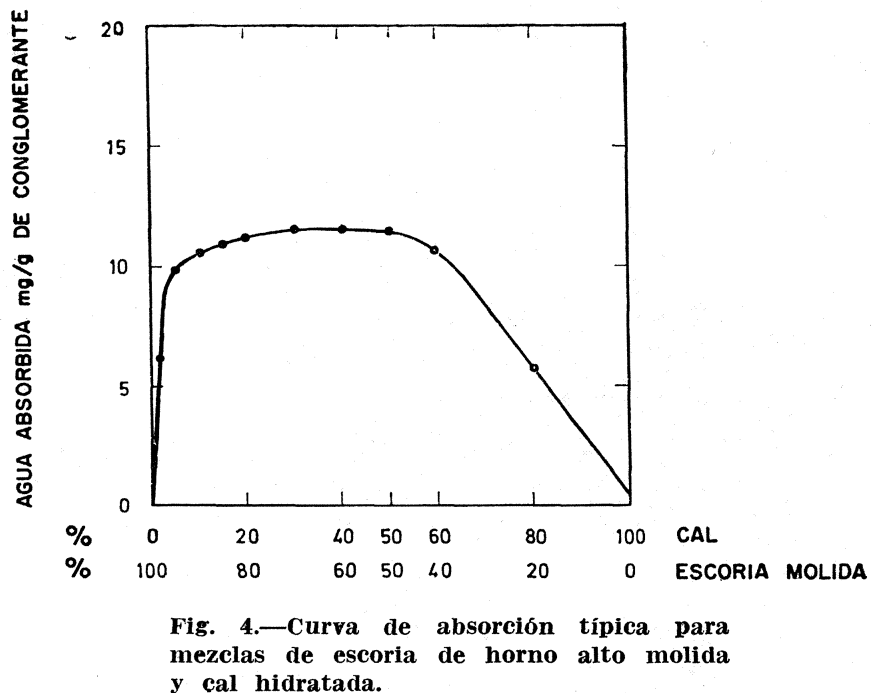

\section{R E F E R E N C I A S}

(1) T. C. Powers and T. L. Brownyard: "Studies of the physical properties of hardened Portland cement pastes" J.A.C.I., Oct. 1946-Apr. 1947, V. 43, P.C.A. Res. Lab. Bull. No. 22.

(2) F. S. Fulton: "The rate of hydration of Portland cement" Portland Cement Institute, Johannesburg, Aug. 1962.

(3) K. SeHLKe: "X-ray diffraction as a method of determining the Portland cement content of mixtures of Portland cement and milled granulated blastfurnace slag" Cement and Lime Manufacture, V. XL, No. 4, July 1967.

(4) J. E. KRUGER: "The use of D.T.A. for estimating the slag content of mixtures of unhydrated Portland cement and ground granulated blastfurnace slag" Cement and Lime Manufacture, V. XXX, No 6, Nov. 1962.

(5) R. E. CRomarty: "The use of a magnetic separator and sulphide determination in the determination of the slag content of Portland blastfurnace cement." Cement and Lime Manufacture, V. XXX,VIII, No. 2, Mar. 1965. 